\title{
Interindividual differences in circadian rhythmicity and sleep homeostasis in older people: effect of a PER3 polymorphism
}

\author{
Antoine U. Viola ${ }^{\mathrm{a}, 1, *}$, Sarah L. Chellappa ${ }^{\mathrm{a}, \mathrm{b}, 1}$, Simon N. Archer ${ }^{\mathrm{c}}$, Fiona Pugin ${ }^{\mathrm{a}}$, \\ Thomas Götz ${ }^{\mathrm{a}}$, Derk-Jan Dijk ${ }^{\mathrm{c}}$, Christian Cajochen ${ }^{\mathrm{a}}$ \\ ${ }^{a}$ Centre for Chronobiology, University of Basel, Basel, Switzerland \\ ${ }^{b}$ The CAPES Foundation/Ministry of Education of Brazil, Brasilia, Brazil \\ ${ }^{c}$ Surrey Sleep Research Centre, University of Surrey, Guildford, UK
}

Received 2 March 2011; received in revised form 20 October 2011; accepted 23 October 2011

\begin{abstract}
Aging is associated with marked changes in the timing, consolidation and structure of sleep. Older people wake up frequently, get up earlier and have less slow wave sleep than young people, although the extent of these age-related changes differs considerably between individuals. Interindividual differences in homeostatic sleep regulation in young volunteers are associated with the variable-number, tandem-repeat (VNTR) polymorphism (rs57875989) in the coding region of the circadian clock gene PERIOD3 (PER3). However, predictors of these interindividual differences have yet to be identified in older people. Sleep electroencephalographic (EEG) characteristics and circadian rhythms were assessed in 26 healthy older volunteers (55-75 years) selected on the basis of homozygosity for either the long or short allele of the PER3 polymorphism. Homozygosity for the longer allele $\left(P E R 3^{5 / 5}\right)$ associated with a phase-advance in the circadian melatonin profile and an earlier occurrence of the melatonin peak within the sleep episode. Furthermore, older $P E R 3^{5 / 5}$ participants accumulated more nocturnal wakefulness, had increased EEG frontal delta activity $(0.75-1.50 \mathrm{~Hz})$, and decreased EEG frontal sigma activity (11-13 Hz) during non-rapid eye movement (REM) sleep compared with $P E R 3^{4 / 4}$ participants. Our results indicate that the polymorphism in the clock gene PER3 may contribute to interindividual differences in sleep and circadian physiology in older people. (C) 2012 Elsevier Inc. All rights reserved.
\end{abstract}

Keywords: Sleep-wake cycle; Circadian rhythms; Constant routine; Melatonin; EEG power density; Clock gene polymorphism; Age

\section{Introduction}

Aging is associated with numerous changes in sleep, such as increased number and duration of awakenings, and reduced time spent in slow wave sleep (SWS), as well as earlier wake-up time (Cajochen et al., 2006; Dijk et al., 1999). The decline in sleep consolidation, together with the advance of sleep timing, may reflect age-related changes in the homeostatic and/or circadian aspects of sleep regulation (Cajochen et al., 2006; Dijk et al., 1999). From the sleep

\footnotetext{
* Corresponding author at: Centre for Chronobiology, Psychiatric Hospital of the University of Basel, Wilhelm Kleinstrasse 27, CH-4012 Basel, Switzerland. Tel.: +41613255318; fax: +41613255556.

E-mail address: antoine.viola@upkbs.ch (A.U. Viola).

${ }^{1}$ Both authors contributed equally to this work.
}

homeostatic perspective, older people display a shallower dissipation of sleep pressure, as indexed by reduced SWS and slow wave activity (SWA) (electroencephalographic [EEG] power density between 0.75 and $4.5 \mathrm{~Hz}$, also referred to as delta activity) dynamics across the night (Dijk and Beersma, 1989; Landolt et al., 1996). From a circadian standpoint, aging can be associated with a reduced circadian amplitude of the core body temperature (CBT) rhythm, and a phase advance of the CBT and melatonin rhythm (Dijk et al., 1999). However, changes in circadian parameters with aging are less consistent than age-related changes of sleep parameters, and SWS in particular. Thus, some (Czeisler et al., 1992; Münch et al., 2005; Van Coevorden et al., 1991; Weitzman et al., 1982), but not all, studies report a decline in the amplitude of CBT, melatonin, and cortisol 
(Monk, 2005; Niggemyer et al., 2004; Zeitzer et al., 1999). The disparity of these findings points to rather large interindividual differences in sleep and circadian rhythmicity with age and leaves the question open as to what is driving these individual differences (Van Cauter et al., 2000). Progress in understanding the genetic and molecular basis of sleep and circadian rhythmicity has led to the identification of genes contributing to interindividual differences in sleep architecture, timing, and duration in humans and mice (Franken and Dijk, 2009; Landolt, 2008; Rétey et al., 2005).

The primate-specific (Jenkins et al., 2005), variablenumber tandem-repeat (VNTR) polymorphism within the coding region of the clock gene PERIOD3 (PER3) contains a 54-nucleotide unit that is repeated $4\left(P E R 3^{4}\right.$ allele $)$ or 5 $\left(P E R 3^{5}\right.$ allele) times in humans (Ebisawa et al., 2001). In young people, the $P E R 3$ VNTR predicts diurnal preference such that those homozygous for the long repeat $\left(P E R 3^{5 / 5}\right)$ prefer earlier wake-up and sleep time (Archer et al., 2003; Lázár et al., in press). Furthermore, in young people, the PER3 VNTR polymorphism also predicts interindividual differences in sleep structure and EEG power density in non-rapid eye movement (REM) sleep, REM sleep and wakefulness (Viola et al., 2007). PER $3^{5 / 5}$ individuals spent more time in SWS, have higher SWA in non-REM sleep, and a steeper dissipation of SWA during sleep episode as compared with those homozygous for the shorter, 4-repeat allele $\left(P E R 3^{4 / 4}\right)$ (Viola et al., 2007). However, in young people, we did not observe significant differences between the genotypes with respect to the phase and amplitude of circadian markers, such as the hormones cortisol and melatonin and the clock genes PER3, BMAL1, and PER2 (Archer et al., 2008; Dijk and Archer, 2010; Viola et al., 2007).

Since the PER3 VNTR polymorphism affects sleep regulation in young individuals, we tested the hypothesis whether this polymorphism also predicts interindividual differences in sleep of older people. Specifically, we predicted that older participants homozygous for the longer repeat $\left(P E R 3^{5 / 5}\right)$ exhibit more slow wave sleep and higher EEG power density in the delta frequency range than older participants homozygous for the shorter repeat $\left(P E R 3^{4 / 4}\right)$.

\section{Methods}

\subsection{Study protocol}

Recruitment of volunteers was conducted through advertisements in newspapers, e-mails, radio, and posted advertisements in the Basel and Alsace areas. The screening procedure began with an interview by telephone or in person, involving a detailed explanation of the study. A total of 168 older men and women (age range: 55-75 years) were recruited, from whom DNA samples and questionnaire data were collected. All participants gave written informed consent. The study protocol, screening questionnaires, and consent forms were approved by the local ethics committee (EKBB, Basel, Switzerland) and conformed to the Decla- ration of Helsinki. All potential study participants were questioned about their sleep quality, life habits, and health state. They completed a consent form, the General Medical Questionnaire, the Short Form-36 (SF-36) Quality of Life Questionnaire, the Horne-Östberg Questionnaire, the $\mathrm{Mu}-$ nich Chronotype Questionnaire, the Pittsburgh Sleep Questionnaire, the Epworth Sleepiness Scale, the Beck Depression Inventory, and the Insomnia Severity Index Questionnaire. Exclusion criteria were medical and sleep disorders, smoking, medication or drug consumption, shift work within the last 3 months, and transmeridian flights during 2 months before the study.

For the genotyping, all potential participants provided buccal swab samples, from which genomic DNA was extracted via the QuickExtract system (Epicentre Biotechnologies, Madison, WI, USA). Genotyping was performed with polymerase chain reaction (PCR) as previously described (Archer et al., 2003) with some modification (Vandewalle et al., 2009).

Volunteers with good general health were included in the field study. Forty participants wore actigraphs (wrist-worn Actiwatch L, Cambridge Neurotechnology, Cambridge, UK) to quantify their rest-activity profile and completed daily sleep diaries for approximately 3 weeks prior to the laboratory study. Complete data were obtained from 37 subjects, out of which 21 were $P E R 3^{5 / 5}$ ( 8 men, 13 women, mean \pm standard error of the mean $[\mathrm{SEM}]=63.02 \pm 1.18$, in years) and 16 were $P E R 3^{4 / 4}$ participants $(8$ men, 8 women, mean $\pm \mathrm{SEM}=63.38 \pm 1.28$, in years). The first 2 weeks were analyzed to characterize the habitual sleepwake cycle for each study participant, who was then instructed to sleep and wake-up according to his/her habitual schedule for 1 week prior to the laboratory study. Compliance was verified by analysis of the sleep diaries and actigraphy data prior to the laboratory study. Participants who successfully completed the 3-week actigraphy underwent a medical examination, blood screening, and a polysomnography recorded adaptation night to exclude sleep disorders. Exclusion criteria were $>10$ periodic leg movements per hour and an apnea-hypopnea index $>10$. Thirteen $P E R 3^{4 / 4}$ individuals and $13 P E R 3^{5 / 5}$ individuals were matched by age, body mass index, gender, and ethnicity and included in the laboratory part of the study. Table 1 describes the main demographic characteristics of these participants, together with data derived from questionnaires and the sleep-wake timings derived from sleep diaries.

\subsection{Laboratory study}

The laboratory part consisted of a baseline sleep episode, followed by approximately 40 hours of extended wakefulness under constant routine (CR) conditions, and a subsequent recovery sleep episode. The CR protocol (Duffy and Dijk, 2002a) was employed to assess the circadian phase and amplitude as well as homeostatic aspects of sleep regulation, which involved 40 hours of extended wakefulness 
Table 1

Demographic and sleep-wake characteristics

\begin{tabular}{lccc}
\hline Genotype & $\begin{array}{l}\text { PER3 } \\
(\mathrm{n}=13)\end{array}$ & $\begin{array}{l}\text { PER3 } \\
(\mathrm{n}=13)\end{array}$ & $p$ \\
\hline Gender, M/F & $5 / 8$ & $5 / 8$ & \\
Age & $62.38 \pm 1.39$ & $62.23 \pm 1.01$ & 0.93 \\
BMI & $25.58 \pm 0.76$ & $24.91 \pm 0.81$ & 0.55 \\
MCTQ & $3.17 \pm 0.21$ & $3.51 \pm 0.17$ & 0.21 \\
PSQI & $3.45 \pm 0.43$ & $3.17 \pm 0.46$ & 0.65 \\
Sleep time & $22: 42 \pm 00: 11$ & $22: 50 \pm 00: 12$ & 0.64 \\
Wake time & $06: 59 \pm 00: 20$ & $07: 05 \pm 00: 11$ & 0.78 \\
Time in bed & $08: 17 \pm 00: 20$ & $08: 15 \pm 00: 16$ & 0.96 \\
HO & $64.22 \pm 2.70$ & $64.00 \pm 2.62$ & 0.95 \\
BDI & $3.89 \pm 0.87$ & $4.00 \pm 1.61$ & 0.95 \\
ESS & $6.44 \pm 0.88$ & $9.40 \pm 1.06$ & 0.04 \\
ISI & $9.67 \pm 2.35$ & $9.40 \pm 2.06$ & 0.93 \\
\hline
\end{tabular}

Sleep time, wake-up time, and time in bed derived from sleep diaries (mean \pm standard error of the mean [SEM]).

Key: BDI, Beck Depression Inventory; BMI, body mass index; ESS, Epworth Sleepiness Scale; F, female; HO, Horne-Östberg Questionnaire; ISI, Insomnia severity index; M, male; MCTQ, Munich Chronotype Questionnaire; PSQI, Pittsburgh Sleep Questionnaire.

under constant environmental conditions (i.e., light $<8$ lux at eye level, temperature approximately $21{ }^{\circ} \mathrm{C}$, regular small isocaloric meals and water, semirecumbent position during scheduled wakefulness, and no information of clock time).

CBT was continuously recorded at 20 -second intervals using an indwelling rectal probe (Interstar, Cham, Switzerland; Therm, type 5500-3, Ahlborn, Holzkirchen, Gemany). The temperature was displayed on a personal computer screen and continuously monitored by a laboratory technician. Hourly saliva samples were collected to measure melatonin and cortisol. All participants received heparin $(0.2 \mathrm{~mL}$ Fragmin, Pfizer, New York NY, USA) at the beginning of the CR and 20 hours afterward, to avoid possible thrombosis. They were attended by technicians throughout the entire CR.

\subsection{Polysomnography measures}

The Vitaport ambulatory system (Vitaport-3 digital recorder, TEMEC Instruments BV, Kerkrade, the Netherlands) was used for all polysomnography recordings. Eight EEG channels (F3, F4, C3, C4, P3, P4, O1, O2) referenced against linked mastoids (A1, A2), 2 electro-oculograms, 2 submental electromyograms, and 2 electrocardiograms were recorded. All EEG signals were low pass filtered at $30 \mathrm{~Hz}$ (fourth order Bessel type antialiasing, total 24 decibels per octave) at a time constant of 1.0 second. After online digitization by using a 12-bit analog-to-digital converter (0.15 $\mathrm{V} / \mathrm{bit}$ ) and a sampling rate at $128 \mathrm{~Hz}$ for the EEG, the raw signals were stored on a Flash RAMCard (Viking, Rancho Santa Margarita, USA) and later downloaded to a PC hard drive. Sleep stages were visually scored per 20-second epochs (Vitaport Paperless Sleep Scoring Software, Kerkrade, the Netherlands) according to standard criteria (Rechtschaffen and Kales, 1968) by a single experienced polysomnography technician, blind to the genotype. Sleep stages were reported as percentages of total sleep time (TST). Sleep latency was defined as latency from lights off to the first epoch of stage 2. Slow wave sleep was defined as the sum of stages 3 and 4 and non-REM sleep as the sum of stages 2,3 , and 4 .

Spectral analysis was conducted using a fast Fourier transform (FFT; 10\% cosine 4-second window) which yielded a $0.25-\mathrm{Hz}$ resolution. To examine EEG power density in the range of $0.75-20 \mathrm{~Hz}$ during non-REM and REM sleep for both genotypes, all-night EEG power density during the baseline and recovery nights was computed for frontal (F3, F4), central (C3, C4), parietal (P3, P4), and occipital $(\mathrm{O} 1, \mathrm{O} 2)$ derivations for each $0.25-\mathrm{Hz}$ frequency bin. To further explore the effects of the 40 hours of extended wakefulness between the genotypes, we computed the difference of recovery and baseline non-REM sleep EEG power density from 0.75 to $20 \mathrm{~Hz}$ in $P E R 3^{4 / 4}$ and $P E R 3^{5 / 5}$ individuals. Data of frontal EEG power density during recovery sleep was excluded for 1 participant due to artifacts across the entire night.

\subsection{Hormonal assays}

Saliva collections for melatonin and cortisol were scheduled every 60 minutes, during the 40 hours of extended wakefulness. A direct double-antibody radioimmunoassay was utilized for the melatonin assay (validated by gas chromatography mass spectroscopy with an analytical least detectable dose of $0.65 \mathrm{pm} / \mathrm{mL}$; Bühlmann Laboratory, Schönenbuch, Switzerland) (Weber et al., 1997). Cortisol was measured by ALPCO (American Laboratory Products Company Diagnostics, Salem, NH, USA), using a direct salivary enzyme-linked immunosorbent assay (ELISA) for quantitative determination of cortisol by enzyme immunoassay. Sensitivity was $1.0 \mathrm{ng} / \mathrm{mL}$ and intra-assay coefficient of variances was $10.3 \%$ for baseline values (b) $6.6 \mathrm{ng} / \mathrm{mL}$.

\subsection{Statistical analysis}

All statistical analyses were performed with SAS version 9.1 (SAS Institute, Cary, NC, USA). To examine EEG power density in the range of $0.75-20 \mathrm{~Hz}$ during non-REM sleep for both genotypes, all-night EEG power density during the baseline and recovery nights was computed for frontal, central, parietal, and occipital derivations for each $0.25-\mathrm{Hz}$ frequency bin. Comparisons between genotypes were made with mixed-model analyses of variance for repeated measures (PROC Mixed), with factors "genotype" $\left(P E R 3^{4 / 4}\right.$ and $\left.P E R 3^{5 / 5}\right)$, "night" (baseline and recovery), "time," and "derivation" (frontal = F3 and F4; central = C3 and $\mathrm{C} 4$; parietal $=\mathrm{P} 3$ and $\mathrm{P} 4$; occipital $=\mathrm{O} 1$ and $\mathrm{O} 2$ ). Contrasts were assessed with the LSMEANS statement. All $p$-values were based on Kenward-Roger's corrected degrees of freedom. To investigate the decay of non-REM EEG delta-power density, we first performed a detection of each quarter $\mathrm{Hz}$ within the delta frequency range $(0.75-4.0 \mathrm{~Hz})$ that had a significant "genotype" effect $(0.75-1.50 \mathrm{~Hz})$. This 
delta range was then utilized to assess the decay of delta activity (on absolute values) for the first 6 hours after sleep onset for EEG of baseline and recovery nights between the genotypes. An exponential decay function (PROC NLIN, SAS 9.1) was fitted as follows: delta $=\operatorname{delta} \infty+\operatorname{delta0} \times \mathrm{e}^{-\mathrm{r} / \mathrm{t}}$, whereby delta $0=$ intercept on y axis, delta $=$ EEG delta power per hour, $r=$ slope of decay, $t=$ time of hour midpoint.

For the analysis of circadian variables such as CBT, melatonin, cortisol, and rest-activity we used a number of approaches. To estimate whether there was an overall effect of genotype, we applied a 2-way r-analysis of variance (ANOVA) with factors "genotype" and "time," the alpha adjustment for multiple comparisons was applied according to Curran-Everett (2000). To estimate the timing and amplitude of rhythmic functions of these circadian variables, we also used a nonlinear regression procedure (PROC NLIN, SAS 9.1), whose function fitted to the data was: Value $\left(\right.$ sample $\left.\mathrm{t}_{\mathrm{i}}\right)=$ Mesor + Amplitude $\times$ sin $(($ sample $\mathrm{t}_{\mathrm{i}}$-phase)/24.2) in which the mesor, amplitude, and phase were free parameters, $t_{i}$ represents the clock time $i$ at which a sample was collected, and value represents the value of the circadian marker variable. Determination of a fitted function for CBT was based on the model: temp $=$ Mesor + Amplitude $\times \sin \left(\left(\right.\right.$ sample $t_{i}$-phase $\left.) / 24.2\right)$ for the analyses of cortisol and melatonin, data were $z$-scored to provide a normalized comparison of amplitudes across these variables. This approach first equates the total variance in each variable in each individual, and then determines how much of this variance can be attributed to a circadian signal. The phase of the melatonin rhythm and cortisol was defined on the basis of the timing of the fitted maximum. In addition, we estimated the phase of the melatonin rhythm, defined as the time of the midpoint between the upward and downward mean crossing. In each individual, the average concentration of melatonin was calculated and the time of the upward and downward crossing of the mean was determined with linear interpolation (Zeitzer et al., 1999). This method of phase determination is robust against individual differences in the amplitude of the melatonin rhythm. Circadian CBT phase was defined as the fitted minimum. The phase angle, or time interval, between habitual wake-up and sleep times and circadian phase was calculated by subtracting the time of fitted melatonin maximum from habitual wake-up and sleep times for each subject (Duffy et al., 2002b).

\section{Results}

\subsection{Demographic and sleep-wake characteristics}

Table 1 summarizes the main demographic and sleepwake characteristics of the $13 P E R 3^{4 / 4}$ and $13 P E R 3^{5 / 5}$ older individuals. Age, body mass index, chronotype, sleep quality, insomnia symptoms, as well as sleep time, wake-up time, and time in bed (derived from the sleep diaries) were typical for healthy older individuals with a relatively higher morningness score. None of these variables differed signif-

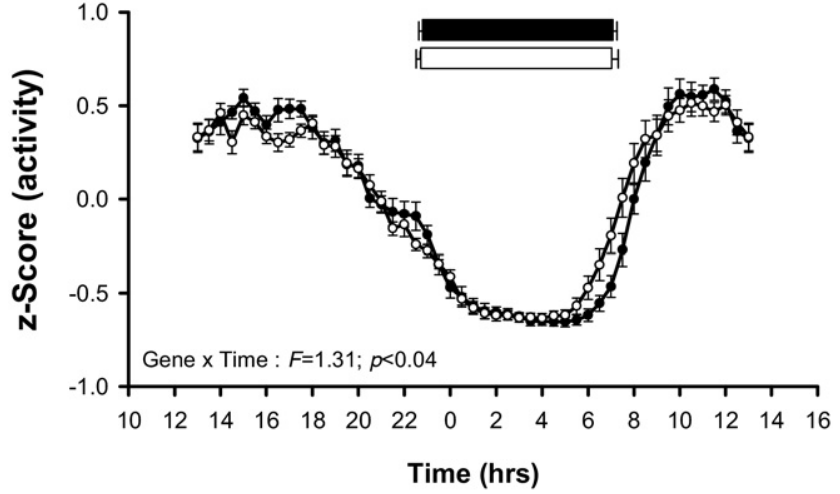

Fig. 1. Double plot of average activity counts of rest-activity cycles quantified by actigraphy (field study) in $21 P E R 3^{5 / 5}$ participants (white circles) and $16 \mathrm{PER}^{4 / 4}$ (black circles). Data are shown as $z$-scores averaged per 20-minute intervals (mean \pm standard error of the mean [SEM]). The time course is plotted relative to time of day (local time).

icantly between the 2 genotypes, except the likelihood of falling asleep during daytime, as assessed by the Epworth Sleepiness scale, which was significantly higher in $P E R 3^{4 / 4}$ than for PER $3^{5 / 5}$ individuals $(p=0.04)$.

\subsection{Circadian markers: rest-activity cycle}

Rest-activity cycles quantified by actigraphy during the field study differed between the genotypes, such that the ANOVA revealed a significant interaction for the factors "genotype" and "time" ( $F=1.31 ; p=0.04)$ (Fig. 1). Furthermore, the assessment of the onset of activity by fitting a rhythmic function to the data revealed a significant difference between the genotypes, such that $P E R 3^{5 / 5}$ participants had an earlier onset of activity, as compared with $P E R 3^{4 / 4}$ participants (mean \pm SEM: PER $3^{5 / 5}=04: 42 \pm 00: 51$ and PER $3^{4 / 4}=$ 06:54 $\pm 00: 25$, in hours; $t$ test; $p=0.04$ ).

\subsection{Circadian markers: CBT, cortisol, and melatonin}

Analyses of the circadian rhythms of CBT and cortisol did not yield statistically significant differences between the genotypes (Fig. 2). By contrast, the analysis of the melatonin profile yielded a significant interaction for the factors "genotype" $\times$ "time" $(F=1.74 ; p=0.011)$. Furthermore, estimation of circadian phase with a nonlinear regression procedure yielded a significant phase advance of the fitted melatonin maximum in the $P E R 3^{5 / 5}$ (mean $\pm \mathrm{SEM}=01$ : $52 \pm 00: 11$, in hours) compared with the $P E R 3^{4 / 4}$ participants (mean \pm SEM $=02: 40 \pm 00: 18$ in hours). These results were corroborated by the significantly earlier melatonin midpoint in the $P E R 3^{5 / 5}$ (mean \pm SEM $=02$ : $18 \pm 00: 15$ in hours) compared with the $P E R 3^{4 / 4}$ volunteers (mean \pm SEM $=03: 40 \pm 00: 33$ in hours; $t$ test; $p=0.03$ ). Analysis of the phase relationship between average wake-up time and timing of melatonin midpoint yielded no significant differences between the genotypes. However, the interval between the average sleep time and melatonin mid- 

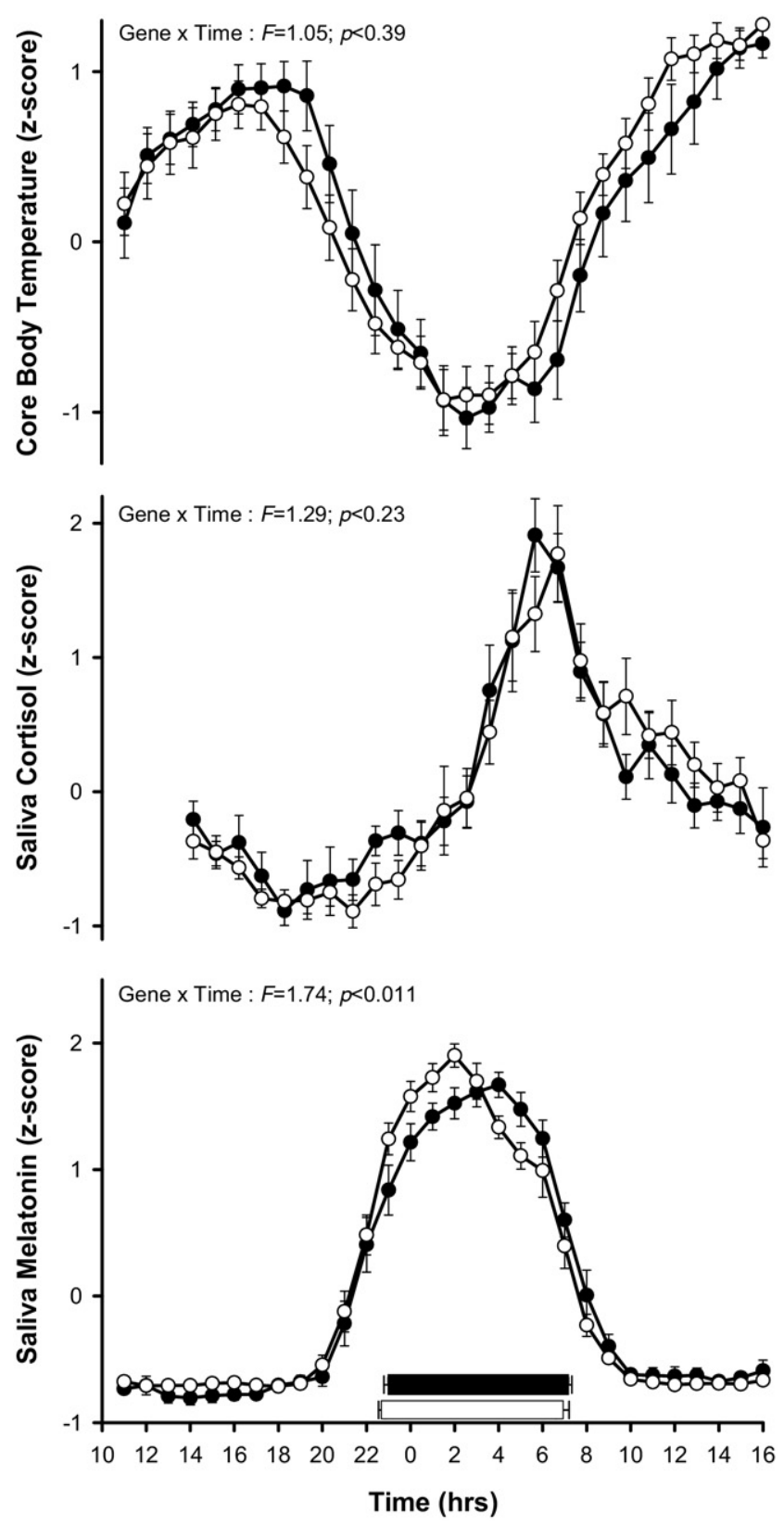

Fig. 2. Time course of core body temperature (top panel), salivary cortisol (middle panel), and salivary melatonin (bottom panel) profiles ( $z$-scores) during 40 hours of extended wakefulness under constant routine conditions in 13 PER $3^{5 / 5}$ participants (white circles) and 13 PER $3^{4 / 4}$ (black circles). Horizontal bars in bottom panel illustrate the timing of the habitual sleep period in $P E R 3^{5 / 5}$ (white bar) and PER $3^{4 / 4}$ (black bar) participants. Data are plotted relative to time of day (local time), and the error bars represent the standard error of the mean.

point was significantly shorter for $P E R 3^{5 / 5}$ (mean \pm SEM $=$ 02:54 $\pm 00: 18$, in hours) than for $P E R 3^{4 / 4}$ participants (mean $\pm \mathrm{SEM}=03: 44 \pm 00: 12$, in hours; $t$ test; $p=0.02$ ).

\subsection{Baseline and recovery sleep structure}

A 2-way r-ANOVA yielded a significant interaction for factors "genotype" $\times$ "night" for total sleep time $(p=$
$0.04)$, and a trend for a significant interaction between these factors for sleep efficiency $(p=0.07)$ and wakefulness $(p=$ 0.06) (Table 2). Main effect "night" was significant for total sleep time, sleep efficiency, non-REM stage 1, and SWS (\% of total sleep time) $(p<0.05)$. When the analyses were restricted to the baseline night, $P E R 3^{5 / 5}$ participants had lower sleep efficiency, and more wakefulness than the PER $3^{4 / 4}$ participants $(p<0.05)$. Analysis of the time course of sleep consolidation, as indexed by accumulated wakefulness, revealed that $P E R 3^{5 / 5}$ participants had significantly more wakefulness during baseline night than $P E R 3^{4 / 4}$ (Wilcoxon 2-sample test $p<0.05$ ), after 4 hours of sleep onset (Fig. 3, left panel). These results were further corroborated by a 2-way r-ANOVA on the number of awakenings (per hour), which yielded a significant "genotype" effect $(F=$ 4.04; $p=0.04$ ) (Fig. 3, right panel).

\subsection{Baseline and recovery sleep EEG power density}

A 3-way r-ANOVA with the factors "genotype," "derivation," and "night" yielded a significant main effect for the factor genotype in the frequency range of 0.75 to $1.50 \mathrm{~Hz}$ (delta range) and of 11 to $13 \mathrm{~Hz}$ (sigma range) $(p<0.05)$. The main effect of "night" was significant in the frequency range of $0.75-10.25 \mathrm{~Hz}$ and $15.50-20 \mathrm{~Hz}(p<0.05)$. The 2 -way interaction of the factors "genotype" $\times$ "derivation" was significant in the frequency range of $0.75-6.5 \mathrm{~Hz}$ and of $8.75-17.25 \mathrm{~Hz}(p<0.05)$. The 2-way interaction "genotype" $\times$ "night" and the 3-way interaction "genotype" $\times$ "night" $\times$ "derivation" yielded no significant differences. Fig. 4 shows that, during the baseline night, $P E R 3^{5 / 5}$ individuals had higher frontal EEG power density in $0.75-1.50$ $\mathrm{Hz}$ (delta range) than the $P E R 3^{4 / 4}$ individuals. Conversely, during the baseline and recovery nights, $P E R 3^{5 / 5}$ individuals had lower frontal EEG power in 11-13 Hz (sigma range) than the $P E R 3^{4 / 4}$ individuals. Furthermore, only during the baseline night, $P E R 3^{5 / 5}$ individuals had higher occipital EEG power density in the frequency range of 5-6 Hz. Analysis of the effects of 40 hours of extended wakefulness (difference of recovery and baseline nights) revealed no significant differences in the response to sleep deprivation in non-REM sleep EEG power density in the frequency range from 0.75 to $20 \mathrm{~Hz}$ between $P E R 3^{4 / 4}$ and $P E R 3^{5 / 5}$ individuals (Fig. 5).

REM sleep EEG power density did not significantly differ between the genotypes during either the baseline or the recovery night (data not shown).

Given the differential levels of the absolute values of delta activity between the genotypes, we computed the exponential decay function (Delta $=$ Delta $\infty+$ Delta $0 \times$ $\mathrm{e}^{-\mathrm{r} / \mathrm{t}}$ ) for frontal non-REM delta activity (by hour), first on an individual basis and then by computing the average parameters for all-night duration of EEG of baseline and recovery nights. A 2-way $\mathrm{r}$-ANOVA yielded a significant interaction for the factors "genotype" $\times$ "time" for delta activity $(F=2.89, p=0.04)$. Homozygosity for the longer 


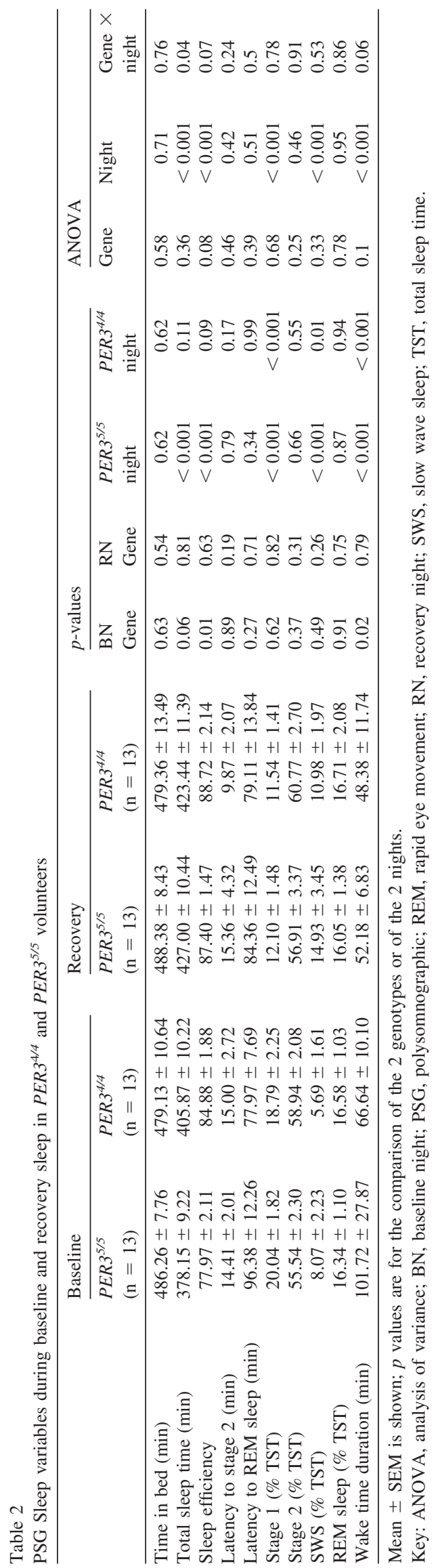

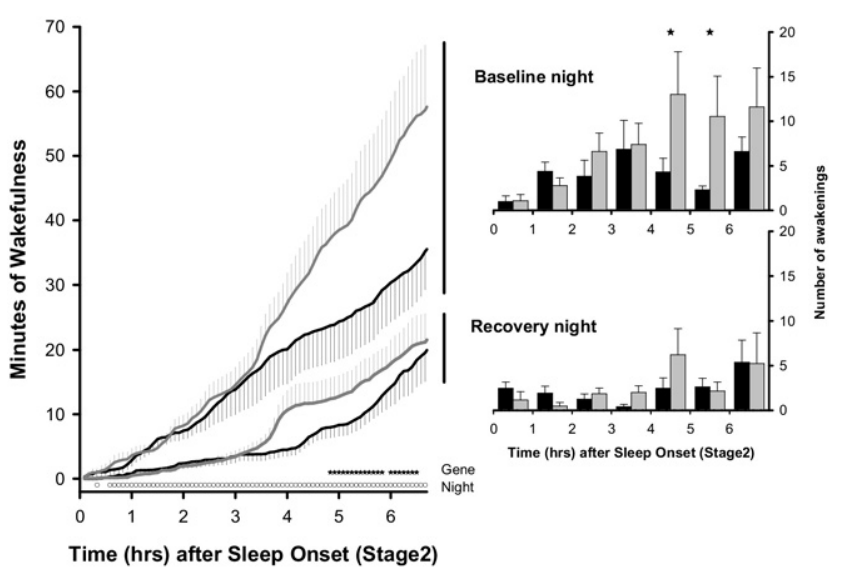

Fig. 3. Accumulation curves for minutes of wakefulness (left panel) after sleep onset (non-rapid eye movement [REM] sleep stage 2) during baseline and recovery nights in $13 P E R 3^{5 / 5}$ (gray lines) and $13 P E R 3^{4 / 4}$ (black lines) participants. Mean \pm standard error of the mean (SEM) values are shown for each 5-minute bin. Horizontal symbols near the abscissa at the bottom indicate the time for which factor "genotype" (stars) and factor "night" (open circles) were significant $(p<0.05)$. Right panel illustrates the number of awakenings (per hour) during baseline and recovery nights in $P E R 3^{5 / 5}$ (gray bars) and $P E R 3^{4 / 4}$ (black bars) participants (mean \pm SEM values; $p<0.05$ ).

allele $\left(P E R 3^{5 / 5}\right)$ was associated with more delta activity in the first 2 hours of the baseline night (first hour: $873.33 \pm 148.34 \mu \mathrm{V}^{2}$; second hour: $762.24 \pm 116.39 \mu \mathrm{V}^{2}$; mean $\pm \mathrm{SEM}$ ) than for $P E R 3^{4 / 4}$ participants (first hour: $577.83 \pm 90.49 \mu \mathrm{V}^{2}$; second hour: $558.44 \pm 77.93 \mu \mathrm{V}^{2}$; mean $\pm \mathrm{SEM} ; t$ test; $p<0.05)$, and a significantly faster dissipation rate $\left(P E R 3^{5 / 5}: 5.2 \pm 1.2\right.$ hours vs. $P E R 3^{4 / 4}$ : $11.6 \pm 2.7$ hours [mean $\pm \mathrm{SEM}$ ]; $t$ test; $p<0.05$ ). After challenging the sleep homeostat by 40 hours of extended wakefulness, delta activity was increased in both the genotypes. However, no significant differences between the genotypes were observed for the amount of delta activity and the rate of dissipation (Fig. 6).

\section{Discussion}

The data show that, in older individuals, the PER3 VNTR polymorphism significantly contributes to individual differences in the rest-activity timing, circadian rhythmicity, and the spectral composition of the EEG during non-REM sleep. Homozygosity for the longer allele $\left(P E R 3^{5 / 5}\right)$ was associated with an earlier activity onset in the morning, an earlier melatonin midpoint, and a shorter interval (phaseangle) between the average sleep time and melatonin midpoint. $P E R 3^{5 / 5}$ participants had reduced sleep efficiency and more wakefulness; only during baseline sleep. PER $3^{5 / 5}$ participants showed increased frontal non-REM delta (0.75$1.50 \mathrm{~Hz})$ and decreased frontal sigma activity $(11-13 \mathrm{~Hz})$ during baseline sleep, while during recovery sleep only frontal sigma activity was decreased. Furthermore, the response to the effects of extended wakefulness was similar 
Baseline

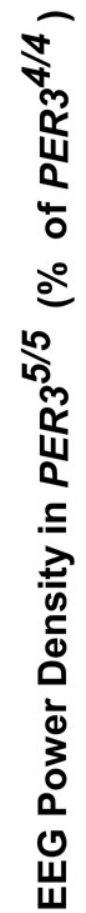
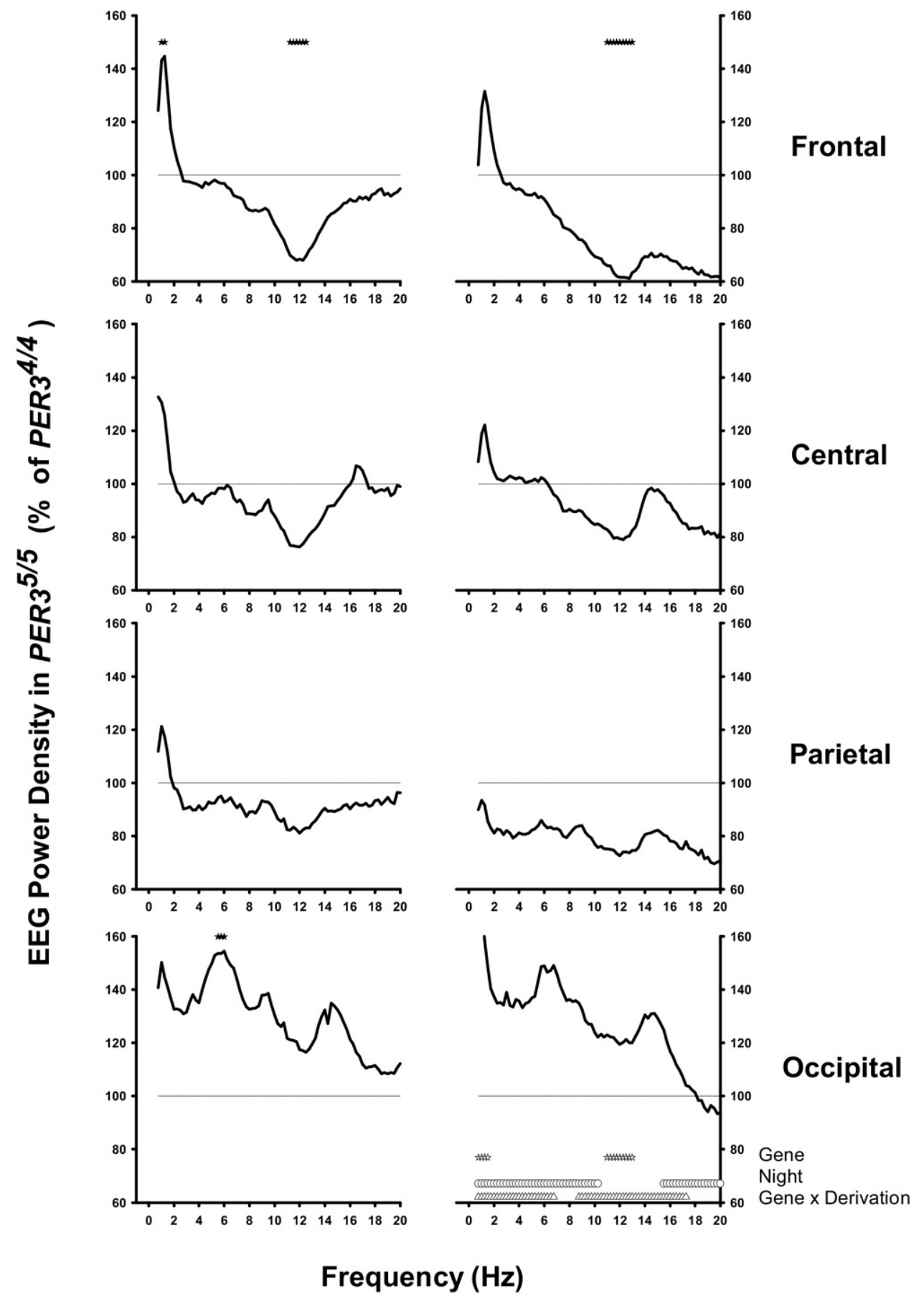

Recovery

Fig. 4. Electroencephalographic (EEG) power density spectra during non-rapid eye movement (REM) sleep in frontal, central, parietal, and occipital derivations during the baseline (left panel) and recovery night (right panel). All-night EEG power density values per 0.25-Hz bin during non-REM sleep in $P E R 3^{5 / 5}$ participants are expressed as percentage of the corresponding average values in PER $3^{4 / 4}$ participants. The horizontal line represents $100 \%$ of EEG power density in the $P E R 3^{4 / 4}$ participants. Mean \pm standard error of the mean (SEM) values are shown for each $0.25-\mathrm{Hz}$ frequency bin in the range of $0.75-20$ Hz. Horizontal symbol near the abscissa at the bottom indicate frequency bins for which factor "genotype" (open stars) and factor "night" (open circles) were significant. Open triangles show frequency bins for which the interaction "genotype" $\times$ "derivation" were significant $(p<0.05)$. Horizontal stars at the top of each panel indicate significant differences between the genotypes for those frequency bins. 


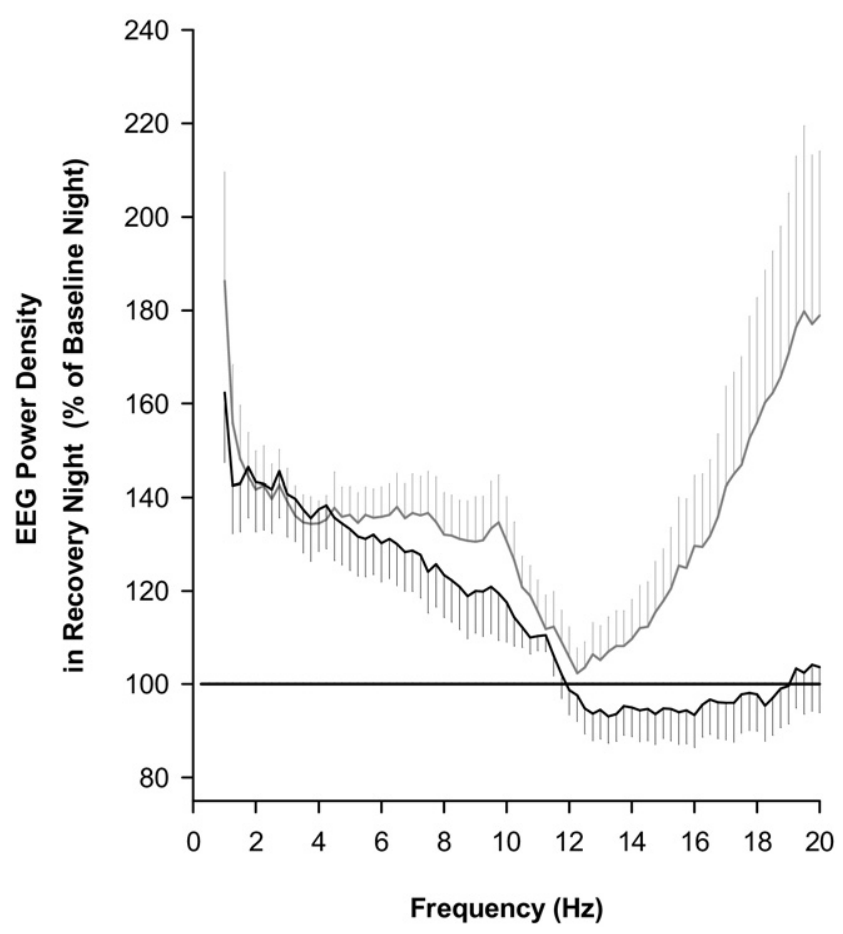

Fig. 5. Electroencephalographic (EEG) non-rapid eye movement (REM) power density in the range of $0.75-20 \mathrm{~Hz}$ during the recovery night in 13 $P E R 3^{5 / 5}$ (gray lines) and $13 P E R 3^{4 / 4}$ (black lines) participants expressed as percentage of EEG power density during baseline (100\%). Data are presented as mean \pm standard error of the mean (SEM).

between the genotypes. Our current findings indicate that in older participants both the circadian and the homeostatic processes seem to be differentially affected by the PER3 VNTR. Thus, we replicated the previous finding in young participants (Viola et al., 2007) that the PER3 polymorphism predicts interindividual differences in sleep (i.e., nonREM delta activity) in an independent sample comprising older participants. However, we did not replicate the increase in visually scored SWS in young $P E R 3^{5 / 5}$, probably due to the very low levels of SWS in older people. The novel aspect of our current study in older people is that the PER3 polymorphism also impacts on the timing of robust circadian markers, such as melatonin.

As expected, the sleep and wake-up times of the current sample of older people was earlier, with higher morningness scores than in the group of young subjects (Viola et al., 2007). The 2 genotype groups were stringently matched for key demographic characteristics, such as age, body mass index (BMI), etc. Thus, it is uncertain how the higher daytime sleepiness score in PER $3^{4 / 4}$ participants relates to the other genotype-dependent changes in sleep and circadian variables. The advance of the melatonin profile and shorter internal phase relationship between the sleep-wake cycle and the melatonin rhythm indicate that $P E R 3^{5 / 5}$ older volunteers slept later relative to the timing of the evening increase in melatonin levels. It remains uncertain if these changes in circadian phase and internal phase relationship in older $P E R 3^{5 / 5}$ volunteers are driven by changes in the period of the circadian oscillator, or differences in light sensitivity or altered light exposure associated with sleep timing. Interestingly, we found no differences with respect to the phase-angle of melatonin and wake-up time. Older participants wake up earlier relative to the morning decline in their melatonin levels (Duffy et al., 2002b) and the nadir of the core body temperature rhythm (Duffy et al., 1998), which suggests that older individuals may be waking up at an earlier circadian phase. Furthermore, changes in the internal phase angle have also been observed in young morning types (Duffy and Czeisler, 2002c) and the PER $3^{5 / 5}$ polymorphism is associated with morningness in young adults (Archer et al., 2003; Lázár et al., in press). Thus, aging and diurnal preference have been associated with changes in the phase angle between sleep and the melatonin rhythm (Dijk and Lockley, 2002), and our results imply that these changes are modulated by the PER3 VNTR polymorphism.

Aging is associated with a reduction of sleep consolidation, with more awakenings in particular from non-REM sleep, which suggests an attenuation of the protective nonREM role for sleep with aging (Dijk et al., 2001). In our study, sleep consolidation was lower in $P E R 3^{5 / 5}$ participants than in $P E R 3^{4 / 4}$ in particular during the second half of the baseline sleep, when homeostatic sleep pressure is low. From a circadian perspective, the reduced sleep consolidation in $P E R 3^{5 / 5}$ older participants may reflect a diminished strength of the circadian drive for sleep.

Sigma activity, which correlates well with sleep spindle activity (Dijk and Czeisler, 1995), was reduced in PER $3^{5 / 5}$ individuals. Sleep spindles comprise the interplay of reticular thalamic, thalamocortical, and cortical pyramidal cells (Steriade et al., 1993), and are under circadian control (Dijk and Czeisler, 1995; Wei et al., 1999), such that the circadian pacemaker actively promotes spindles during the night, presumably to promote sleep consolidation (Dijk and Czeisler, 1995). Sleep spindles have been described as inhibitory events that can predict sleep stability (Dang-Vu et al., 2010), and both its frequency and its circadian modulation can decline with age (Dijk and Czeisler, 1995; Knoblauch et al., 2005; Wei et al., 1999). Furthermore, the circadian modulation of sleep spindle characteristics varies with brain location, such that the major difference is between frontal and parietal spindle frequency activity (Cajochen and Dijk, 2003). Frontal spindles were found to have a lower frequency (approximately $12 \mathrm{~Hz}$ ) than parietal spindles (approximately $14 \mathrm{~Hz}$ ). The observation that our genotype dependent changes in spindle activity were observed for a lower frequency range (11-13 Hz), and that these differences were most apparent in frontal brain areas, may indicate that the age-related decrease in sigma activity is modulated by the PER3 VNTR, presumably through changes in the circadian promotion of spindle activity.

Homozygosity for the longer allele $\left(P E R 3^{5 / 5}\right)$ affected baseline EEG power density, with increased non-REM fron- 
Baseline

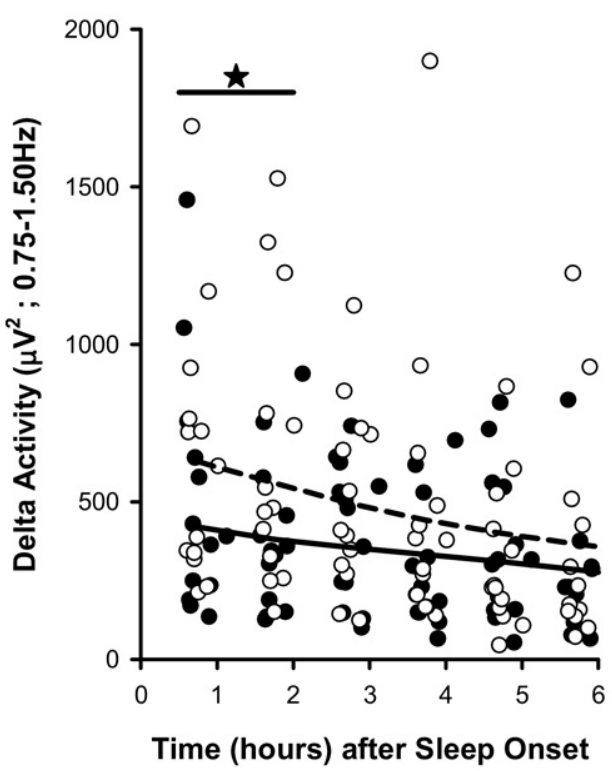

Recovery

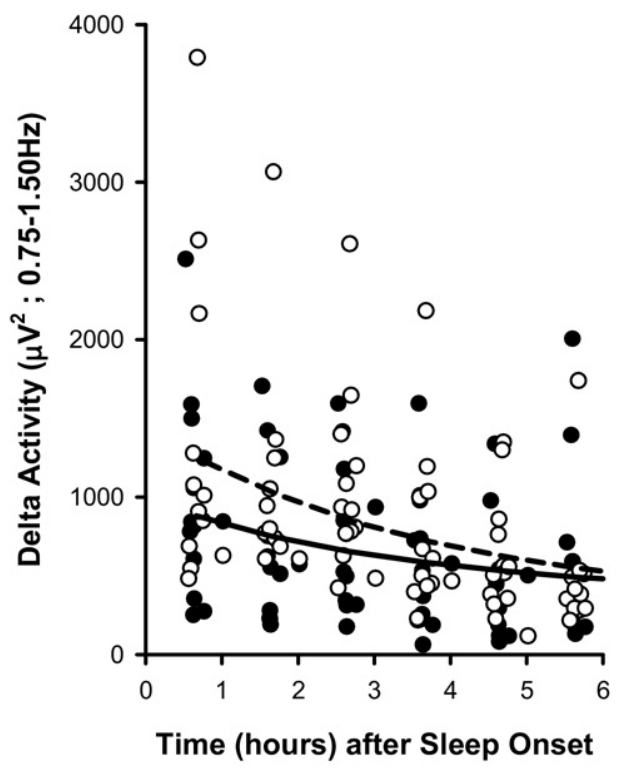

Fig. 6. Time course of frontal absolute delta activity during baseline (left panel) and recovery (right panel) nights. Lines represent an exponential decay function (delta $=$ delta $\infty+$ delta0 $\times \mathrm{e}^{- \text {rt }}$ ) of non-rapid eye movement (REM) electroencephalographic (EEG) delta power $(0.75-1.5 \mathrm{~Hz})$ fitted to the data separately for the 2 genotypes. Dashed lines and white circles: PER $3^{5 / 5}$ participants; solid lines and black circles: PER $3^{4 / 4}$ participants. Data are plotted by time (in hours) after sleep onset (non-REM sleep stage 2). * Significant difference between genotypes for the amount of absolute values of delta activity.

tal delta activity. At the beginning of sleep, delta activity, particularly in frontal derivations, acts as a functional index of increased homeostatic sleep pressure (Borbély et al., 1981; Cajochen et al., 2001). Thus, our findings indicate that, as for young individuals, the dynamics of the sleep homeostatic process also differ between older $P E R 3^{5 / 5}$ and $P E R 3^{4 / 4}$ individuals. Furthermore, in the absence of sleep deprivation, $P E R 3^{5 / 5}$ individuals initiate sleep at a higher homeostatic level and display a faster dissipation of this sleep pressure. The lower dissipation rate in delta activity observed in our older participants is in accordance with previous observations and is largely independent of topography (Münch et al., 2004). Interestingly, very large interindividual differences in the dissipation slope were observed for the $P E R 3^{4 / 4}$ but not for $P E R 3^{5 / 5}$ participants, particularly after sleep deprivation. Whether or not this is related to other differences between the genotypes remains unknown.

The topographical distribution of delta activity is such that, during both sleep and wakefulness, the homeostatic increase in low-EEG components is most prominent in frontal brain areas (Cajochen et al., 1999; Finelli et al., 2000), indicating higher susceptibility to the effects of sleep deprivation. Our results on frontal delta activity indicate that $P E R 3^{5 / 5}$ may experience higher sleep pressure, as compared with $P E R 3^{4 / 4}$ participants, and that the frontal cortex is the most susceptible to the effects of this higher sleep pressure. With respect to the occipital derivation, to our knowledge there are no previous studies that have shown such frequency-unspecific changes in this derivation with advanced age. Thus, the reason as to why we observed higher EEG power density in $P E R 3^{5 / 5}$ participants (across all frequency ranges) remains elusive. Aging affects both the homeostatic and the circadian aspects of sleep, and the present data seem to indicate that these age-related changes are more prominent in $P E R 3^{5 / 5}$ older volunteers. Our study sample did not include young $P E R 3^{5 / 5}$ and $P E R 3^{4 / 4}$ volunteers, thus we could not conduct a direct age-related comparison for the homeostatic and circadian changes. Furthermore, even a direct comparison would only constitute a cross-sectional comparison and not establish whether the PER3 VNTR interacts with the aging process per se, as this would require a longitudinal study. The present data show that key aspects of age-related changes in sleep and circadian rhythmicity, such as earlier melatonin phase, shorter phase-angle, reduced sigma activity, and sleep consolidation, are more pronounced in $P E R 3^{5 / 5}$ older volunteers. Thus, we speculate that $P E R 3^{5 / 5}$ individuals show more distinct age-related changes commonly seen in sleep structure and circadian phase. These data have implications for our understanding of the interindividual differences in agerelated changes in sleep and circadian rhythmicity.

\section{Disclosure statement}

The authors disclose no conflicts of interest related to this study.

The study protocol, screening questionnaires, and consent form were approved by the local Ethical Committee and conformed to the Declaration of Helsinki. 


\section{Acknowledgements}

We thank Claudia Renz, Marie-France Dattler, and Giovanni Balestrieri for their help in data acquisition. This research was supported by the Velux Foundation $\left(n^{\circ} 449\right.$, Switzerland).

\section{References}

Archer, S.N., Robilliard, D.L., Skene, D.J., Smits, M., Williams, A., Arendt, J., von Schantz, M., 2003. A length polymorphism in the circadian clock gene Per3 is linked to delayed sleep phase syndrome and extreme diurnal preference. Sleep 26, 413-415.

Archer, S.N., Viola, A.U., Kyriakopoulou, V., Von Schantz, M., Dijk, D., 2008. Inter-Individual differences in habitual sleep timing and entrained phase of endogenous circadian rhythms of BMAL1, PER2 and PER3 mRNA in human leucocytes. Sleep 5, 608-617.

Borbély, A.A., Baumann, F., Brandeis, D., Strauch, I., Lehmann, D., 1981. Sleep deprivation: effect on sleep stages and EEG power density in man. Electroencephalogr. Clin. Neurophysiol. 51, 483-493.

Cajochen, C., Dijk, D.J., 2003. Electroencephalographic activity during wakefulness, rapid eye movement, and non-rapid-eye movement sleep in humans: comparison of their circadian and homeostatic modulation. Sleep Biol. Rhythms 2, 85-95.

Cajochen, C., Foy, R., Dijk, D.J., 1999. Frontal predominance of a relative increase in sleep delta and theta EEG activity after sleep loss in humans. Sleep Res. Online 2, 65-69.

Cajochen, C., Knoblauch, V., Kräuchi, K., Renz, C., Wirz-Justice, A., 2001. Dynamics of frontal EEG activity, sleepiness and body temperature under high and low sleep pressure. Neuroreport 12, 22772281.

Cajochen, C., Münch, M., Knoblauch, V., Blatter, K., Wirz-Justice, A., 2006. Age-related changes in the circadian and homeostatic regulation of human sleep. Chronobiol. Int. 23, 1-14.

Curran-Everett, D., 2000. Multiple comparisons: philosophies and illustrations. Am. J. Physiol. Regul. Integr. Comp. Physiol. 279, R1-R8.

Czeisler, C.A., Dumont, M., Duffy, J.F., Steinberg, J.D., Richardson, G.S., Brown, E.N., Sánchez, R., Ríos, C.D., Ronda, J.M., 1992. Association of sleep-wake habits in older people with changes in output of circadian pacemaker. Lancet 340, 933-936.

Dang-Vu, T.T., McKinney, S.M., Buxton, O.M., Solet, J.M., Ellenbogen, J.M., 2010. Spontaneous brain rhythms predict sleep stability in the face of noise. Curr. Biol. 20, R626-R627.

Dijk, D.J., Archer, S.N., 2010. PERIOD3, circadian phenotypes, and sleep homeostasis. Sleep Med. Rev. 14, 151-160.

Dijk, D.J., Beersma, D.G., van den Hoofdakker, R.H., 1989. All night spectral analysis of EEG sleep in young adult and middle-aged male subjects. Neurobiol. Aging 10, 677-682.

Dijk, D.J., Czeisler, C.A., 1995. Contribution of the circadian pacemaker and the sleep homeostat to sleep propensity, sleep structure, electroencephalographic slow waves, and sleep spindle activity in humans. J. Neurosci. 15, 3526-3538.

Dijk, D.J., Duffy, J.F., Czeisler, C.A., 2001. Age-related increase in awakenings: impaired consolidation of nonREM sleep at all circadian phases. Sleep 24, 565-577.

Dijk, D.J., Duffy, J.F., Riel, E., Shanahan, T.L., Czeisler, C.A., 1999. Ageing and the circadian and homeostatic regulation of human sleep during forced desynchrony of rest, melatonin and temperature rhythms. J. Physiol. 516, 611-627.

Dijk, D.J., Lockley, S.W., 2002. Integration of human sleep-wake regulation and circadian rhythmicity. J. Appl. Physiol. 92, 852-862.

Duffy, J.F., Czeisler, C.A., 2002c. Age-related change in the relationship between circadian period, circadian phase, and diurnal preference in humans. Neurosci. Lett. 318, 117-120.
Duffy, J.F., Dijk, D.J., 2002a. Getting through to circadian oscillators: why use constant routines? J. Biol. Rhythms 17, 4-13.

Duffy, J.F., Dijk, D.J., Klerman, E.B., Czeisler, C.A., 1998. Later endogenous circadian temperature nadir relative to an earlier wake time in older people. Am. J. Physiol. 275, R1478-R1487.

Duffy, J.F., Zeitzer, J.M., Rimmer, D.W., Klerman, E.B., Dijk, D.J., Czeisler, C.A., 2002b. Peak of circadian melatonin rhythm occurs later within the sleep of older subjects. Am. J. Physiol. Endocrinol. Metab. 282, E297-E303.

Ebisawa, T., Uchiyama, M., Kajimura, N., Mishima, K., Kamei, Y., Katoh, M., Watanabe, T., Sekimoto, M., Shibui, K., Kim, K., Kudo, Y., Ozeki, Y., Sugishita, M., Toyoshima, R., Inoue, Y., Yamada, N., Nagase, T., Ozaki, N., Ohara, O., Ishida, N., Okawa, M., Takahashi, K., Yamauchi, T., 2001. Association of structural polymorphisms in the human period3 gene with delayed sleep phase syndrome. EMBO Rep. 2, 342-346.

Finelli, L.A., Baumann, H., Borbély, A.A., Achermann, P., 2000. Dual electroencephalogram markers of human sleep homeostasis: correlation between theta activity in waking and slow-wave activity in sleep. Neuroscience 101, 523-529.

Franken, P., Dijk, D.J., 2009. Circadian clock genes and sleep homeostasis. Eur. J. Neurosci. 29, 1820-1829.

Jenkins, A., Archer, S.N., von Schantz, M., 2005. Expansion during primate radiation of a variable number tandem repeat in the coding region of the circadian clock gene Period3. J. Biol. Rhythms 20, 470-472.

Knoblauch, V., Münch, M., Blatter, K., Martens, W.L., Schröder, C., Schnitzler, C., Wirz-Justice, A., Cajochen, C., 2005. Age-related changes in the circadian modulation of sleep-spindle frequency during nap sleep.Sleep 28, 1093-1101.

Landolt, H.P., 2008. Genotype-dependent differences in sleep, vigilance, and response to stimulants. Curr. Pharm. Des. 14, 3396-3407.

Landolt, H.P., Dijk, D.J., Achermann, P., Borbély, A.A., 1996. Effect of age on the sleep EEG: slow-wave activity and spindle frequency activity in young and middle-aged men. Brain Res. 738, 205-212.

Lázár, A.S., Slak, A., Lo, J., Santhi, N., von Schantz, M., Archer, S.N., Groeger, J.A., Dijk, D.J., in press. Sleep, diurnal preference, health and psychological well-being: a prospective single-allelic variation study. Chronobiol Int.

Monk, T.H., 2005. Aging human circadian rhythms: conventional wisdom may not always be right. J. Biol. Rhythms 20, 366-374.

Münch, M., Knoblauch, V., Blatter, K., Schröder, C., Schnitzler, C., Kräuchi, K., Wirz-Justice, A., Cajochen, C., 2004. The frontal predominance in human EEG delta activity after sleep loss decreases with age. Eur. J. Neurosci. 20, 1402-1410.

Münch, M., Knoblauch, V.,Blatter, K., Schröder, C., Schnitzler, C.,Kräuchi, K., Wirz-Justice, A., Cajochen, C.,2005. Age-related attenuation of the evening circadian arousal signal in humans. Neurobiol. Aging 26, 1307-1319.

Niggemyer, K.A., Begley, A., Monk, T., Buysse, D.J., 2004. Circadian and homeostatic modulation of sleep in older adults during a 90-minute day study. Sleep 27, 1535-1541.

Rechtschaffen, A., Kales, A., 1968. A manual of standardized terminology, techniques and scoring system for sleep stages of human subjects. US Dept of Health, Education and Welfare, Public Health Service, Bethesda, MD.

Rétey, J.V., Adam, M., Honegger, E., Khatami, R., Luhmann, U.F., Jung, H.H., Berger, W., Landolt, H.P., 2005. A functional genetic variation of adenosine deaminase affects the duration and intensity of deep sleep in humans. Proc. Natl. Acad. Sci. U. S. A. 102, 15676-15681.

Steriade, M., McCormick, D.A., Sejnowski, T.J., 1993. Thalamocortical oscillations in the sleeping and aroused brain. Science 262, 679-685.

Van Cauter, E., Leproult, R., Plat, L., 2000. Age-related changes in slow wave sleep and REM sleep and relationship with growth hormone and cortisol levels in healthy men. JAMA 284, 861-868.

Van Coevorden, A., Mockel, J., Laurent, E., Kerkhofs, M., L'HermiteBalèriaux, M., Decoster, C., Néve, P., Van Cauter, E., 1991. Neuroendocrine rhythms and sleep in aging men. Am. J. Physiol. Regul. Integr. Comp. Physiol. 260, E651-E661.

Vandewalle, G., Archer, S.N., Wuillaume, C., Balteau, E., Degueldre, C., Luxen, A., Maquet, P., Dijk, D.J., 2009. Functional magnetic resonance 
imaging-assessed brain responses during an executive task depend on interaction of sleep homeostasis, circadian phase, and PER3 genotype. J. Neurosci. 29, 7948-7956.

Viola, A.U., Archer, S.N., James, L.M., Groeger, J.A., Lo, J.C., Skene, D.J., Von Schantz, M., Dijk, D.J., 2007. PER3 polymorphism predicts sleep structure and waking performance. Curr. Biol. 17, 613618.

Weber, J.M., Schwander, J.C., Unger, I., Meier, D., 1997. A direct ultrasensitive RIA for the determination of melatonin in human saliva: comparison with serum levels. J. Sleep Res. 26, 757.
Wei, H.G., Riel, E., Czeisler, C.A., Dijk, D.J., 1999. Attenuated amplitude of circadian and sleep-dependent modulation of electroencephalographic sleep spindle characteristics in elderly human subjects. Neurosci. Lett. 260, 29-32.

Weitzman, E.D., Moline, M.L., Czeisler, C.A., Zimmerman, J.C., 1982. Chronobiology of aging: temperature, sleep-wake rhythms and entrainment. Neurobiol. Aging 3, 299-309.

Zeitzer, J.M., Daniels, J.E., Duffy, J.F., Klerman, E.B., Shanahan, T.L., Dijk, D.J., Czeisler, C.A., 1999. Do plasma melatonin concentrations decline with age? Am. J. Med. 107, 432-436. 